\title{
Path integral duality and Planck scale corrections to the primordial spectrum in exponential inflation
}

\author{
L. Sriramkumar* \\ Harish-Chandra Research Institute, Chhatnag Road, Jhunsi, Allahabad 211 019, India. \\ S. Shankaranarayanan ${ }^{\dagger}$ \\ HEP Group, International Centre for Theoretical Physics, Strada costiera 11, 34100 Trieste, Italy \\ Max-Planck-Institut für Gravitationsphysik, Albert-Einstein-Institut, Am Mühlenberg 1, D-14476 Potsdam, Germany
}

(Dated: August 1, 2018)

\begin{abstract}
The enormous red-shifting of the modes during the inflationary epoch suggests that physics at the Planck scale may modify the standard, nearly, scale-invariant, primordial, density perturbation spectrum. Under the principle of path-integral duality, the space-time behaves as though it has a minimal length $L_{\mathrm{P}}$ (which we shall assume to be of the order of the Planck length), a feature that is expected to arise when the quantum gravitational effects on the matter fields have been taken into account. Using the method of path integral duality, in this work, we evaluate the Planck scale corrections to the spectrum of density perturbations in the case of exponential inflation. We find that the amplitude of the corrections is of the order of $\left(\mathcal{H} / M_{\mathrm{P}}\right)$, where $\mathcal{H}$ and $M_{\mathrm{P}}$ denote the inflationary and the Planck energy scales, respectively. We also find that the corrections turn out to be completely independent of scale. We briefly discuss the implications of our result, and also comment on how it compares with an earlier result.
\end{abstract}

PACS numbers: $98.80 . \mathrm{Cq}, 04.62 .+\mathrm{v}$

\section{INTRODUCTION}

The inflationary scenario $[1,2]$ provides an attractive mechanism to generate the primordial density perturbations that are eventually responsible for the anisotropies in the cosmic microwave background (CMB) and the formation of the large scale structure (LSS). Typically, the CMB and LSS data have been used to constrain the parameters of the inflationary model such as, for example, the height and the slope of the canonical scalar field potential $[3,4]$. However, over the last few years, it has been realized that, in most of the models of inflation [5], the period of acceleration lasts so long that length scales that are of cosmological interest today would have emerged from sub-Planckian length scales at the beginning of inflation. In other words, inflation provides a magnifying glass to probe physics at the very high energy scales (say, $\sim 10^{17} \mathrm{GeV}$ ) that are otherwise inaccessible to observations or experiments. This has led to a considerable effort in the literature towards understanding the effects of Planck scale physics on the inflationary perturbation spectrum [6]-[16], and the resulting signatures on the CMB [17-20].

In an exponential (i.e. de Sitter) or a power-law inflationary scenario, the spectrum of perturbations is essentially given by the Fourier transform of the Wightman function of a free, massless and minimally coupled scalar field (see, for instance, Ref. [2]). Therefore, in order to study the effects of Planck scale physics on the primor-

\footnotetext{
*E-mail:sriram@mri.ernet.in

${ }^{\dagger}$ E-mail:shanki@aei.mpg.de
}

dial spectrum, we need to understand as to how quantum gravitational effects modify the two-point function of a scalar field in an inflationary background. But, due to the lack of a viable quantum theory of gravitation, one is forced to consider phenomenological models constructed by hand-models which are supposed to contain one or more features of the actual effective theory obtained by integrating out the gravitational degrees of freedom. The different high energy models that have been considered in this context modify either the dynamics or the initial conditions (and, in some cases, both) of the canonical scalar field [7]-[16].

A class of models that has been used extensively to study the Planck scale corrections to the primordial spectrum involves the violation of local Lorentz invariance at the energy scales of inflation [7, 8]. However, theoretically, there exists no apriori reason to believe that Lorentz invariance may be broken at the inflationary energy scale. Moreover, certain observations and experiments seem to indicate that local Lorentz invariance may be preserved to extremely high energies (see Ref. [21]; in this context, also see Ref. [22]). In such a situation, to study the Planck scale effects, it becomes important to consider models that preserve local Lorentz invariance even as they contain a fundamental scale [14].

One such model that introduces a fundamental length scale while preserving Lorentz invariance is the approach due to the principle of path-integral duality [23]. Recall that, in standard quantum field theory, the path integral amplitude for a path connecting events $\tilde{x}$ and $\tilde{x}^{\prime}$ in a given space-time is proportional to the proper length, say, $\mathcal{R}\left(\tilde{x}, \tilde{x}^{\prime}\right)$ between the two events. The duality principle proposes that the path integral amplitude should be invariant under the transformation $\mathcal{R} \rightarrow\left(L_{\mathrm{p}}^{2} / \mathcal{R}\right)$, 
where $L_{\mathrm{P}}$ is assumed to be of the order of the Planck length. A consequence of this postulate is that, in the two-point function in the Minkowski vacuum, the proper distance, say, $(\Delta \tilde{x})^{2}$ between two space-time events is replaced by $\left[(\Delta \tilde{x})^{2}+4 L_{\mathrm{P}}^{2}\right]$. This suggests that, under the duality principle, the background space-time behaves as though it possesses a minimal length $L_{\mathrm{P}}$, a feature that is expected to arise when the quantum gravitational effects are taken into account.

In this work, we shall utilize the locally Lorentz invariant approach due to path integral duality to evaluate the Planck scale corrections to the spectrum of density perturbations in the exponential inflationary scenario. It should be pointed out here that, recently, another approach that is based on T-duality in string theory has been used to evaluate the Planck scale corrections to the standard spectrum of gravitational waves in exponential inflation [24]. There seem to exist some correspondence between the approaches of T-duality in string theory and the principle of path integral duality ${ }^{1}$. However, we would like to emphasize here the following crucial difference between the approach motivated by T-duality in string theory that has been adopted in Ref. [24] and the approach due to path integral duality we shall adopt. In Ref. [24], the authors use a modified dispersion relation to mimic the effects due to T-duality on the propagator. Clearly, such an approach assumes violation of local Lorentz invariance. In this work, we shall instead use the proper time representation of the modified propagator [23] - an approach that explicitly preserves local Lorentz invariance - to evaluate the Planck scale corrections to the spectrum of perturbations. As we shall see, the amplitude of the corrections prove to be the order of $\left(\mathcal{H} / M_{\mathrm{P}}\right)$, where $\mathcal{H}$ and $M_{\mathrm{P}}$ denote the inflationary and the Planck energy scales. Moreover, in contrast to the results obtained in Ref. [24], we find that the corrections turn out to be completely independent of scale.

The remainder of this paper is organized as follows. The modifications to the two-point functions that arise due to the path integral duality are easily expressed in terms of the Schwinger's proper time representation for the Greens functions [23]. Therefore, in the following section, using the proper time method, we shall very briefly outline as to how the approach of path integral duality modifies the two-point functions of a quantum scalar field propagating in a curved space-time. We shall also point out the initial conditions that need to be imposed for evaluating the perturbation spectrum through the proper time method. In Section III, we shall obtain the standard, scale invariant perturbation spectrum in exponential inflation through the proper time formalism. In Section IV, we shall evaluate the Planck scale correc-

\footnotetext{
${ }^{1}$ For instance, using the T-duality in string theory, it has been shown that the propagator for the string center of mass results in the same propagator as obtained through the principle of path integral duality. For details, see Ref. [25].
}

tions to the scale invariant spectrum using the approach of path integral duality. Finally, in Section V, we conclude with a summary and discussion of the results we have obtained.

A few words on our convention and notation are in order at this stage of our discussion. The metric signature we shall adopt is $(+,-,-,-)$, and we shall set $\hbar=c=1$. Also, for convenience, we shall denote the set of four coordinates $x^{\mu}$ as $\tilde{x}$.

\section{PATH INTEGRAL DUALITY MODIFIED TWO-POINT FUNCTIONS}

Consider a free and minimally coupled scalar field of mass $m$ that is propagating in a classical gravitational background described by the metric tensor $g_{\mu \nu}$. In Schwinger's proper time formalism, the Feynman Green's function corresponding to such a scalar field can be expressed as $[26,27]$

$$
G_{\mathrm{F}}\left(\tilde{x}, \tilde{x}^{\prime}\right)=i \int_{0}^{\infty} d s e^{-i m^{2} s} K\left(\tilde{x}, \tilde{x}^{\prime} ; s\right)
$$

where $K\left(\tilde{x}, \tilde{x}^{\prime} ; s\right)$ is defined as

$$
K\left(\tilde{x}, \tilde{x}^{\prime} ; s\right) \equiv\left\langle\tilde{x}\left|e^{-i \boxminus s}\right| \tilde{x}^{\prime}\right\rangle .
$$

In other words, the quantity $K\left(\tilde{x}, \tilde{x}^{\prime} ; s\right)$ is the path integral amplitude for a quantum mechanical system described by the following Hamiltonian:

$$
\widehat{\mathbf{H}}=\triangle \equiv \frac{1}{\sqrt{-g}} \partial_{\mu}\left(g^{\mu \nu} \partial_{\nu}\right)
$$

It can be shown that demanding the principle of path integral duality corresponds to modifying the above expression for the Feynman propagator to (for details, see Refs. [23])

$$
G_{\mathrm{F}}^{(\mathrm{M})}\left(\tilde{x}, \tilde{x}^{\prime}\right)=i \int_{0}^{\infty} d s e^{i L_{\mathrm{P}}^{2} / s} e^{-i m^{2} s} K\left(\tilde{x}, \tilde{x}^{\prime} ; s\right),
$$

where, as pointed out earlier, $L_{\mathrm{P}}$ is of the order of the Planck length. We shall utilize this prescription to evaluate the Planck scale corrections to the inflationary perturbation spectrum. However, before proceeding with the calculations, we need to clarify two technical issues.

Firstly, in cosmological perturbation theory, the spectra of perturbations are determined by the Fourier transform of the Hadamard function (i.e. the symmetric twopoint function) of the quantum field. The two-point function (1), however, is a Feynman Green's function rather than the Hadamard function. It can be shown that, for a minimally coupled scalar field, the Hadamard function 
can be written $a^{2}$

$$
G^{(1)}\left(\tilde{x}, \tilde{x}^{\prime}\right)=\int_{-\infty}^{\infty} d s e^{-i m^{2} s} K\left(\tilde{x}, \tilde{x}^{\prime} ; s\right)
$$

where $K\left(\tilde{x}, \tilde{x}^{\prime} ; s\right)$ is the kernel defined in Eq. (2). Therefore, the path integral duality modified Hadamard function can be written as

$$
G_{(\mathrm{M})}^{(1)}\left(\tilde{x}, \tilde{x}^{\prime}\right)=\int_{-\infty}^{\infty} d s e^{i L_{\mathrm{P}}^{2} / s} e^{-i m^{2} s} K\left(\tilde{x}, \tilde{x}^{\prime} ; s\right) .
$$

Secondly, in a time-dependent background, since the field evolves with time, the states at two different instants are, in general, different. In the inflationary scenario, the perturbations are evaluated as expectation values in the in-vacuum, a state which is defined when the modes are well inside the Hubble radius (see our discussion immediately before and after Eq. (8) below). Therefore, when evaluating the kernel $K\left(\tilde{x}, \tilde{x}^{\prime} ; s\right)$, we need to impose suitable initial conditions to ensure that the resulting Green function is an in-in function (rather than the in-out function that is usually considered in, say, S-matrix or scattering calculations).

In the next section, we shall obtain the scale invariant perturbation spectrum in a de Sitter universe using the expression (5) for the Hadamard function. And, in Section IV, we shall evaluate the Planck scale corrections to the scale invariant spectrum using the modified Hadamard function (6).

\section{PERTURBATION SPECTRUM THROUGH THE PROPER TIME METHOD}

As we had mentioned in the introduction, in an exponential inflationary scenario, the metric perturbations (both scalar and tensor perturbations) can be described by a massless and minimally coupled scalar field, say, $\Phi$, which satisfies the following equation of motion $[1,2]$ :

$$
\square \Phi=0 .
$$

Also, the perturbations are assumed to be induced by the fluctuations in the free quantum field $\hat{\Phi}$. Therefore, the power spectrum as well as the statistical properties of the perturbations are completely characterized by the twopoint functions of the quantum field. The power spectrum of the scalar perturbations per logarithmic interval,

\footnotetext{
2 See Appendix A. In this appendix, we explicitly illustrate this result for the case of the Hadamard function in the Minkowski vacuum.
}

viz. $\left[k^{3} \mathcal{P}_{\Phi}(k)\right]$, is defined as $[1,2]$

$$
\begin{aligned}
\int_{0}^{\infty}\left(\frac{d k}{k}\right)\left[k^{3} \mathcal{P}_{\Phi}(k)\right] & =\left\langle 0\left|\hat{\Phi}^{2}(\eta, \mathbf{x})\right| 0\right\rangle \\
& =G^{+}(\tilde{x}, \tilde{x})=\left(\frac{1}{2}\right) G^{(1)}(\tilde{x}, \tilde{x}),
\end{aligned}
$$

where $|0\rangle$ is the vacuum state of the field, and $G^{+}\left(\tilde{x}, \tilde{x}^{\prime}\right)$ and $G^{(1)}\left(\tilde{x}, \tilde{x}^{\prime}\right)$ denote the Wightman and the Hadamard functions of the quantum field, respectively.

Consider a spatially-flat Friedmann universe described by the line-element

$$
d s^{2}=d t^{2}-a^{2}(t) d \mathbf{x}^{2},
$$

where $t$ is the cosmic time and $a(t)$ denotes the scale factor. The symmetry of the Friedmann background allows us to write the kernel $K\left(\tilde{x}, \tilde{x}^{\prime} ; s\right)$ as follows (see, for example, Ref. [28]):

$$
K\left(\tilde{x}, \tilde{x}^{\prime} ; s\right)=\int \frac{d^{3} \mathbf{k}}{(2 \pi)^{3}} e^{i \mathbf{k} \cdot\left(\mathbf{x}-\mathbf{x}^{\prime}\right)}\left\langle t\left|e^{i \hat{\mathrm{H}}_{k} s}\right| t^{\prime}\right\rangle,
$$

where

$$
\hat{\mathrm{H}}_{k} \equiv-\left(\frac{1}{a^{3}}\right) \frac{d}{d t}\left(a^{3} \frac{d}{d t}\right)-\left(\frac{k^{2}}{a^{2}}\right) .
$$

Therefore, for a massless scalar field, the Hadamard function (5) is given by

$$
G^{(1)}\left(\tilde{x}, \tilde{x}^{\prime}\right)=\int_{-\infty}^{\infty} d s \int \frac{d^{3} \mathbf{k}}{(2 \pi)^{3}} e^{i \mathbf{k} \cdot\left(\mathbf{x}-\mathbf{x}^{\prime}\right)}\left\langle t\left|e^{i \hat{\mathrm{H}}_{k} s}\right| t^{\prime}\right\rangle .
$$

From this expression and the definition (8) of the power spectrum, we then obtain

$$
\begin{aligned}
{\left[k^{3} \mathcal{P}_{\Phi}(k)\right] } & =\left(\frac{k^{3}}{4 \pi^{2}}\right) \int_{-\infty}^{\infty} d s\left\langle t\left|e^{i \hat{\mathrm{H}}_{k} s}\right| t\right\rangle \\
& =\left(\frac{k^{3}}{4 \pi^{2}}\right) \int_{-\infty}^{\infty} d s\left\langle t\left|e^{-i \hat{\mathrm{H}}_{k}(-s)}\right| t\right\rangle .
\end{aligned}
$$

The Schrodinger equation corresponding to the Hamiltonian $\hat{\mathrm{H}}_{k}$ is given by

$$
\hat{\mathrm{H}}_{k} \psi_{E} \equiv-\left(\frac{1}{a^{3}}\right) \frac{d}{d t}\left(a^{3} \frac{d \psi_{E}}{d t}\right)-\left(\frac{k^{2}}{a^{2}}\right) \psi_{E}=E \psi_{E},
$$

where the wave function $\psi_{E}$ is normalized as follows:

$$
\int_{\text {all } t} d t a^{3}(t) \psi_{E}(t) \psi_{E^{\prime}}^{*}(t)=\delta\left(E-E^{\prime}\right) .
$$

(In the de Sitter background that we are interested in, the energy $E$ turns out to be a continuous distribution. 
Hence we have normalized the wave functions using the delta function normalization.) If we write

$$
\psi_{E}(t)=a^{-3 / 2}(t) \chi_{E}(t),
$$

then we find that the function $\chi_{E}(t)$ satisfies the differential equation

$$
\begin{array}{r}
-\left(\frac{d^{2} \chi_{E}}{d t^{2}}\right)-\left[\left(\frac{k^{2}}{a^{2}}\right)-\left(\frac{3}{4}\right)\left(\frac{\dot{a}}{a}\right)^{2}-\left(\frac{3}{2}\right)\left(\frac{\ddot{a}}{a}\right)\right] \chi_{E} \\
=E \chi_{E},
\end{array}
$$

and the normalization condition (15) reduces to

$$
\int_{\text {all } t} d t \chi_{E}(t) \chi_{E^{\prime}}^{*}(t)=\delta\left(E-E^{\prime}\right) .
$$

On using the Feynman-Kac formula (see, for instance, Refs. $[29,30])$, we can write the kernel $\left\langle t\left|e^{-i \hat{H}_{k}(-s)}\right| t\right\rangle$ in terms of the wave function $\chi_{E}$ as follows:

$$
\begin{aligned}
\left\langle t\left|e^{-i \hat{\mathrm{H}}_{k}(-s)}\right| t\right\rangle & =\int_{\text {all } E} d E\left|\psi_{E}(t)\right|^{2} e^{i E s} \\
& =\left(\frac{1}{a^{3}(t)}\right) \int_{\text {all } E} d E\left|\chi_{E}(t)\right|^{2} e^{i E s},
\end{aligned}
$$

where the integral is all over the values of the energy $E$. Substituting this expression in the expression (13) for the power spectrum, we obtain that

$$
\left[k^{3} \mathcal{P}_{\Phi}(k)\right]=\left(\frac{k^{3}}{4 \pi^{2} a^{3}(t)}\right) \int_{-\infty}^{\infty} d s \int_{\text {all } E} d E\left|\chi_{E}(t)\right|^{2} e^{i E s} .
$$

On interchanging the order of the integrals and carrying the integral over $s$ first, we find that the power spectrum reduces to

$$
\begin{aligned}
{\left[k^{3} \mathcal{P}_{\Phi}(k)\right] } & =\left(\frac{k^{3}}{2 \pi a^{3}(t)}\right) \int_{\text {all } E} d E \delta(E)\left|\chi_{E}(t)\right|^{2} \\
& =\left(\frac{k^{3}}{2 \pi a^{3}(t)}\right)\left|\chi_{0}(t)\right|^{2} .
\end{aligned}
$$

Note that the integral over $s$ leads to a 'density of states' that is a delta function in $E$. Evidently, this implies that only the wave function corresponding to $E=0$ contributes to the sum, thereby leading to the standard result one usually arrives at by the canonical quantization procedure. As we shall see later, path integral duality introduces Planck scale modifications to the 'density of states', and these modifications in turn lead to the quantum gravitational corrections in the power spectrum.

We shall now use the above procedure to calculate the power spectrum of the perturbations in de Sitter inflation. Consider the case of exponential inflation described by the scale factor

$$
a(t)=e^{\mathcal{H} t},
$$

where $\mathcal{H}$ denotes the energy scale during inflation. In such a background, the Schrodinger equation (17) reduces to

$$
-\left(\frac{d^{2} \chi_{E}}{d t^{2}}\right)-\left[k^{2} e^{-2 \mathcal{H} t}-\left(\frac{9 \mathcal{H}^{2}}{4}\right)\right] \chi_{E}=E \chi_{E} .
$$

The general solution to this differential equation is given by

$$
\chi_{E}(t)=N_{E} H_{\nu}^{(1)}\left(k \mathcal{H}^{-1} e^{-\mathcal{H} t}\right)+M_{E} H_{\nu}^{(2)}\left(k \mathcal{H}^{-1} e^{-\mathcal{H} t}\right),
$$

where $N_{E}$ and $M_{E}$ are $E$-dependent constants which are determined by the initial conditions and the normalization condition (18). The functions $H_{\nu}^{(1)}$ and $H_{\nu}^{(2)}$ are the Hankel functions of the first and the second kinds, respectively, and the quantity $\nu$ is given by

$$
\nu=\left[\left(\frac{9}{4}\right)-\left(\frac{E}{\mathcal{H}^{2}}\right)\right]^{1 / 2} .
$$

In inflationary cosmology, the initial conditions are imposed at very early times when the modes are well within the Hubble radius. Also, it is assumed that, in the subHubble limit (i.e. when $(k / a) \gg \mathcal{H}$ ), the modes are in the vacuum state. In the Schrodinger picture that we are working with here, this condition corresponds to choosing $\chi_{E}$ to be an 'outgoing' wave at the 'left infinity'. For the case of de Sitter, this condition reduces to

$$
\lim _{t \rightarrow-\infty} \chi_{E}(t) \propto \exp -\left(i k \mathcal{H}^{-1} e^{-\mathcal{H} t}\right)
$$

which can be achieved by setting condition $M_{E}$ to zero in Eq. (23). The quantity $N_{E}$ can be determined using the normalization condition (18). We find that it is given by (for details, see Appendix B)

$$
N_{E}= \begin{cases}{\left[\frac{|\sin (\pi \nu)|}{4 \mathcal{H}}\right]^{1 / 2},} & \text { for } E<\left(9 \mathcal{H}^{2} / 4\right), \\ {\left[\frac{\sinh (\pi|\nu|) e^{-(2 \pi|\nu|)}}{4 \mathcal{H}}\right]^{1 / 2},} & \text { for } E \geq\left(9 \mathcal{H}^{2} / 4\right),\end{cases}
$$

so that we have

$$
\chi_{E}(t)=\left\{\begin{array}{r}
{\left[\frac{|\sin (\pi \nu)|}{4 \mathcal{H}}\right]^{1 / 2} H_{\nu}^{(1)}\left(k \mathcal{H}^{-1} e^{-\mathcal{H} t}\right),} \\
\text { for } E<\left(9 \mathcal{H}^{2} / 4\right), \\
{\left[\frac{\sinh (\pi|\nu|) e^{-(2 \pi|\nu|)}}{4 \mathcal{H}}\right]^{1 / 2} H_{i|\nu|}^{(1)}\left(k \mathcal{H}^{-1} e^{-\mathcal{H} t}\right)} \\
\text { for } E \geq\left(9 \mathcal{H}^{2} / 4\right) .
\end{array}\right.
$$

The wave function for $E=0$ is then given by

$$
\chi_{0}(t)=\left(\frac{1}{\sqrt{4 \mathcal{H}}}\right) H_{(3 / 2)}^{(1)}\left(k \mathcal{H}^{-1} e^{-\mathcal{H} t}\right)
$$

and the power spectrum (20) can be written as

$$
\left[k^{3} \mathcal{P}_{\Phi}(k)\right]=\left(\frac{k^{3}}{8 \pi \mathcal{H} e^{-3 \mathcal{H} t}}\right)\left|H_{(3 / 2)}^{(1)}\left(k \mathcal{H}^{-1} e^{-\mathcal{H} t}\right)\right|^{2} .
$$


Using the expression for the Hankel function $H_{(3 / 2)}^{(1)}$ (see, for instance, Refs. [1]) it is then straightforward to obtain the following spectrum at Hubble exit [i.e. when $\left.\left(k \mathcal{H}^{-1} e^{-\mathcal{H} t}\right)=1\right]$ :

$$
\left[k^{3} \mathcal{P}_{\Phi}(k)\right]=\left(\frac{\mathcal{H}^{2}}{2 \pi^{2}}\right)
$$

which is the standard, scale-invariant, spectrum one obtains in de Sitter. It should be pointed out here that, in obtaining the above expression, we have evaluated the power spectrum when the modes leave the Hubble radius. In the literature, the spectrum of perturbations is usually evaluated at the super-Hubble scales. In the standard theory, the power spectra at Hubble exit and at super-Hubble scales typically differ in amplitude by a factor of order unity.

\section{PLANCK SCALE CORRECTIONS DUE TO PATH INTEGRAL DUALITY}

In this section, using the approach of path integral duality, we shall evaluate the Planck scale corrections to the scale invariant perturbation spectrum (30).

To obtain the modified power spectrum, we shall use the path integral duality modified Hadamard function (6) in the definition (8) of the power spectrum. Using the expression (10) for the complete kernel, and the FeynmanKac formula (19) for the kernel in the time direction, it is straightforward to show that the modified spectrum is given by

$$
\begin{aligned}
{\left[k^{3} \mathcal{P}_{\Phi}(k)\right]_{(\mathrm{M})}=} & \left(\frac{k^{3}}{4 \pi^{2} a^{3}(t)}\right) \int_{-\infty}^{\infty} d s e^{i L_{\mathrm{P}}^{2} / s} \\
& \times \int_{\text {all } E} d E\left|\chi_{E}(t)\right|^{2} e^{i E s} \\
= & \left(\frac{k^{3}}{4 \pi^{2} a^{3}(t)}\right) \int_{\text {all } E} d E\left|\chi_{E}(t)\right|^{2} \\
& \times \int_{-\infty}^{\infty} d s e^{i\left[\left(L_{\mathrm{P}}^{2} / s\right)+E s\right]}
\end{aligned}
$$

where, in the second expression, as we had done earlier, we have interchanged the order of integration over $E$ and $s$. On carrying out the integral over $s$, we obtain the path integral duality modified 'density of states' to be (for details, see Appendix C):

$$
\begin{aligned}
\int_{-\infty}^{\infty} d s & e^{i\left[\left(L_{\mathrm{P}}^{2} / s\right)+E s\right]} \\
& =(2 \pi)\left[\delta(E)-\theta(E)\left(\frac{L_{P}}{\sqrt{E}}\right) J_{1}\left(2 L_{\mathrm{P}} \sqrt{E}\right)\right],
\end{aligned}
$$

where $\theta(E)$ denotes the step function and $J_{1}$ is the Bessel function of order unity. On substituting this 'density of states' in the expression (31), we obtain the modified power spectrum to be

$$
\begin{aligned}
{\left[k^{3} \mathcal{P}_{\Phi}(k)\right]_{(\mathrm{M})} } & \left(\frac{k^{3}}{2 \pi a^{3}(t)}\right)\left|\chi_{0}(t)\right|^{2} \\
= & -\left(\frac{L_{\mathrm{P}} k^{3}}{2 \pi a^{3}(t)}\right) \int_{0}^{\infty} \frac{d E}{\sqrt{E}} J_{1}\left(2 L_{\mathrm{P}} \sqrt{E}\right)\left|\chi_{E}(t)\right|^{2}
\end{aligned}
$$

The following points need to be emphasized regarding the expressions we have obtained above for the modified 'density of states' and the corresponding power spectrum $^{3}$. It is clear that it is the second term in these expressions that leads to the Planck scale corrections in the 'density of states' and the primordial spectrum. Importantly, as required, this additional term vanishes in the limit of $L_{\mathrm{P}} \rightarrow 0$ so that we recover the standard result.

Before we proceed to evaluate the corrections, it is important that we highlight another feature of the correction term in the above modified spectrum, and make suitable clarifying remarks. Note that, in the expression (33) for the modified spectrum, it is wave functions with energy $E>0$ that contribute to the corrections. In field theoretic language, wave functions with $E>0$ correspond to massive modes. (Recall that the first term in (33) arises due to the $E=0$, which corresponds to a massless mode, in confirmation of the canonical picture.) In other words, it is the massive modes that lead to the Planck scale corrections and, in fact, in our model, the corrections are a sum of the contributions due to all the massive modes. But, it is well known that, in the standard inflationary picture, the amplitude of massive modes decays at super-Hubble scales. Hence, within the conventional scenario, the amplitude of the corrections would be expected to decay at the super-Hubble scales. However, we shall adopt the point of view that, since, in our model, the massive modes represent the Planck scale corrections to the standard, massless modes, the mechanism that 'freezes' the amplitude of the standard spectrum at super Hubble scales will also 'freeze' the amplitude of the Planck scale corrections at their value at Hubble exit. Therefore, in what follows, we shall evaluate the corrections to the standard power spectrum when the modes leave the Hubble radius.

Let us now evaluate the Planck scale corrections to the spectrum (30) we had obtained in the last section for the case of the de Sitter universe. On substituting the wave function (27) in the expression (33) for the modified

\footnotetext{
3 We should stress here that the quantity in Eq. (32) is not positive definite and, therefore, it does not represent a genuine density of states. We have used the term 'density of states' simply as a convenient shorthand for referring to this quantity.
} 
power spectrum and imposing the condition of Hubble exit, we obtain that

$$
\left[k^{3} \mathcal{P}_{\Phi}(k)\right]_{(\mathrm{M})}=\left(\frac{\mathcal{H}^{2}}{4 \pi^{2}}\right)-\mathcal{C}\left(k, \mathcal{H}, L_{\mathrm{P}}\right),
$$

where the term $\mathcal{C}\left(k, H, L_{\mathrm{P}}\right)$ denotes the Planck scale corrections to the scale invariant power spectrum and is given by

$$
\begin{aligned}
& \mathcal{C}\left(k, \mathcal{H}, L_{\mathrm{P}}\right) \\
&=\left(\frac{\mathcal{H}^{2} L_{\mathrm{P}}}{8 \pi}\right) \int_{0}^{\left(9 \mathcal{H}^{2} / 4\right)} \frac{d E}{\sqrt{E}} J_{1}\left(2 L_{\mathrm{P}} \sqrt{E}\right) \sin (\pi \nu) \\
& \times\left|H_{\nu}^{(1)}(1)\right|^{2} \\
&+\left(\frac{\mathcal{H}^{2} L_{\mathrm{P}}}{8 \pi}\right) \int_{\left(9 \mathcal{H}^{2} / 4\right)}^{\infty} \frac{d E}{\sqrt{E}} J_{1}\left(2 L_{\mathrm{P}} \sqrt{E}\right) \sinh (\pi|\nu|) \\
& \times e^{-(2 \pi|\nu|)}\left|H_{\nu}^{(1)}(1)\right|^{2} .
\end{aligned}
$$

Clearly, the corrections are independent of $k$. Therefore, the corrections do not result in any features in the standard, scale invariant power spectrum, but they simply change the amplitude of the spectrum ${ }^{4}$. In fact, in a de Sitter universe, this feature seems to be common to many of the high energy models that have been considered in the literature - for example, see, Refs. [13, 34]. Since the amplitude cannot be determined from the observations, the above corrections are, in principle, unobservable. Nevertheless, the amplitude of the Planck scale corrections is of theoretical interest and, in what follows, we shall evaluate this amplitude.

In terms of the variable

$$
\mathcal{E}=\left(2 L_{\mathrm{P}} \sqrt{E}\right)
$$

we find that the corrections $\mathcal{C}\left(k, \mathcal{H}, L_{\mathrm{P}}\right)$ can be written as

$$
\mathcal{C}\left(k, \mathcal{H}, L_{\mathrm{P}}\right)=\left(\frac{\mathcal{H}^{2}}{8 \pi}\right)[\mathcal{A}(\delta)+\mathcal{B}(\delta)]
$$

where the quantities $\mathcal{A}$ and $\mathcal{B}$ depend only on the dimensionless quantity $\delta$ defined to be

$$
\delta=\left(\mathcal{H} L_{\mathrm{P}}\right)=\left(\mathcal{H} / M_{\mathrm{P}}\right)
$$

with $M_{\mathrm{P}}$ being the Planck mass. The quantities $\mathcal{A}$ and

\footnotetext{
${ }^{4}$ In Appendix D, as a consistency check, we have explicitly shown that, in the limit of $H \rightarrow 0$, the modified spectrum evaluated at a given time reduces to the expected power spectrum of a scalar field in flat space-time.
}

$\mathcal{B}$ are described by the integrals

$$
\begin{aligned}
& \mathcal{A}(\delta)=\int_{0}^{(3 \delta)} d \mathcal{E} J_{1}(\mathcal{E}) \sin (\pi \nu)\left|H_{\nu}^{(1)}(1)\right|^{2} \\
& \mathcal{B}(\delta)=\int_{(3 \delta)}^{\infty} d \mathcal{E} J_{1}(\mathcal{E}) \sinh (\pi|\nu|) e^{-(2 \pi|\nu|)}\left|H_{\nu}^{(1)}(1)\right|^{2}
\end{aligned}
$$

and the quantity $\nu$ can be expressed in terms of $\mathcal{E}$ and $\delta$ as follows:

$$
\nu=\left[\left(\frac{9}{4}\right)-\left(\frac{\mathcal{E}}{2 \delta}\right)^{2}\right]^{1 / 2} .
$$

However, closed form expressions for the above integrals describing $\mathcal{A}$ and $\mathcal{B}$ do not seem to exist. Therefore, we have evaluated them numerically and, in the figure below, we have plotted the sum of $\mathcal{A}$ and $\mathcal{B}$ as a function of $\delta$. It is evident from the above figure that, in a de

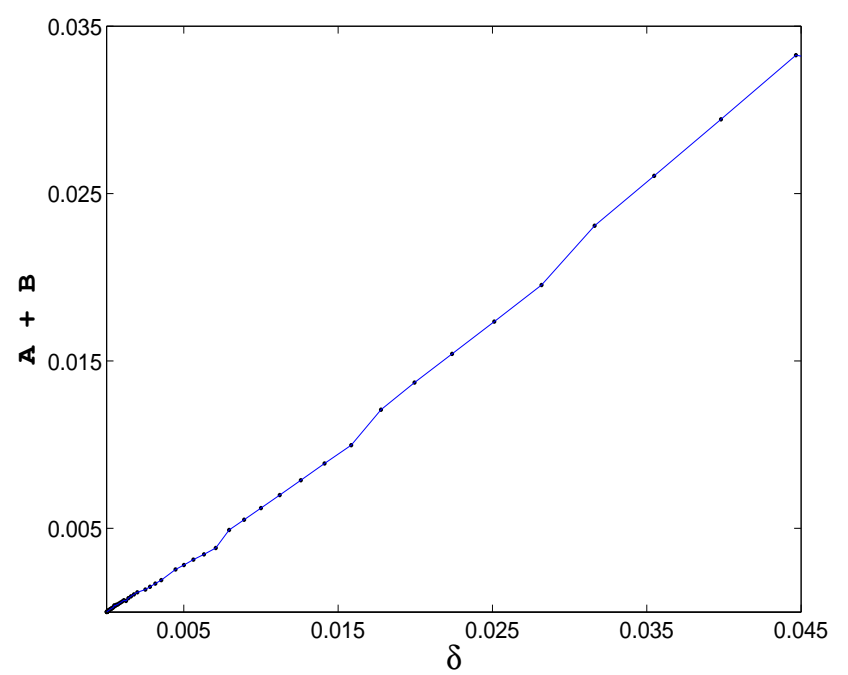

FIG. 1: $(\mathcal{A}+\mathcal{B})$ plotted as a function of the quantity $\delta$.

Sitter universe, the Planck scale corrections to primordial spectrum are linear in $\delta$.

\section{SUMMARY AND DISCUSSION}

In this work, using the locally Lorentz invariant approach of path integral duality, we have evaluated the Planck scale corrections to the primordial perturbation spectrum in the case of exponential inflation. We find that the Planck scale corrections are of the order of $\left(\mathcal{H} / M_{\mathrm{P}}\right)$. Moreover, the corrections are completely scalefree, which implies that they simply change the amplitude of the power spectrum. As we pointed out in the last section, the amplitude in itself cannot be determined from the observations and, as a result, the corrections we 
have obtained are unobservable. However, the amplitude of the Planck scale corrections (whether they would depend on, say, $\left(\mathcal{H} / M_{\mathrm{P}}\right)$ or $\left.\left(\mathcal{H} / M_{\mathrm{P}}\right)^{2}\right)$ have been of theoretical interest and, our calculation assumes importance in this context.

At this stage, it is interesting to compare our results with that of Ref. [24]. As we had mentioned in the introduction, in this work, the authors evaluate the power spectrum of the gravitational waves in exponential inflation. For computational convenience, they use a particular modified dispersion relation to mimic the high energy effects. For the case of the minimal energy initial statewhich is similar to the vacuum state we have chosen in Eq. (25) - they obtain a spectrum which has a blue tilt with superimposed oscillations. In contrast, using the locally Lorentz invariant proper time method, we obtain a spectrum that is strictly scale invariant with the Planck scale corrections of order $\left(\mathcal{H} / M_{\mathrm{P}}\right)$.

In cosmological perturbation theory, it is well-known that, the metric perturbations do not couple to the matter perturbations in a de Sitter background (see, for instance, Ref. [2]). For this reason, the exact de Sitter case is considered pathological and, ideally, one would like to carry out the analysis in slow-roll inflation, or, at least for the case of power-law inflationary scenario. However, the wave functions $\chi_{E}$ for $E \neq 0$ are not known in an analytic form in such cases and one needs to resort to a WKB approximation to evaluate the wave functions and the resulting corrections. We hope to address this issue in a forthcoming publication.

\section{Acknowledgments}

The authors would wish to thank T. Padmanabhan for discussions and comments on the manuscript. LS would like to thank the High Energy, Cosmology and Astroparticle Physics Group of The Abdus Salam International Centre for Theoretical Physics, Trieste, Italy for hospitality, where part of this work was carried out.

\section{APPENDIX A: PROPER TIME REPRESENTATION OF THE HADAMARD FUNCTION}

In this appendix, we shall outline as how the Hadamard function in flat space-time can be written as in Eq. (16). Let us begin by recalling that the Hadamard function of a quantized, scalar field $\hat{\Phi}$ is defined as follows:

$$
G^{(1)}\left(\tilde{x}, \tilde{x}^{\prime}\right)=\left\langle 0\left|\hat{\Phi}(\tilde{x}) \hat{\Phi}\left(\tilde{x}^{\prime}\right)+\hat{\Phi}\left(\tilde{x}^{\prime}\right) \hat{\Phi}(\tilde{x})\right| 0\right\rangle .
$$

Consider a massive, scalar field in flat space-time. Using the canonical quantization procedure, it is straightfor- ward to show that the Hadamard function is given by

$$
\begin{array}{r}
G^{(1)}\left(\tilde{x}, \tilde{x}^{\prime}\right)=\int \frac{d^{3} \mathbf{k}}{(2 \pi)^{3}}\left(\frac{1}{2 \omega}\right)\left(e^{-i \omega\left(t-t^{\prime}\right)}+e^{i \omega\left(t-t^{\prime}\right)}\right) \\
\times e^{i \mathbf{k} \cdot\left(\mathbf{x}-\mathbf{x}^{\prime}\right)},
\end{array}
$$

where $\omega^{2}=\left(k^{2}+m^{2}\right)$. This expression can, in turn, be written as

$$
\begin{aligned}
G^{(1)}\left(\tilde{x}, \tilde{x}^{\prime}\right) & =\int \frac{d^{3} \mathbf{k}}{(2 \pi)^{3}} \int \frac{d k_{0}}{\left(2 k_{0}\right)}[ \\
& \left.\delta\left(k_{0}-\omega\right)+\delta\left(k_{0}+\omega\right)\right] \\
& \times e^{-i\left[k_{0}\left(t-t^{\prime}\right)-\mathbf{k} \cdot\left(\mathbf{x}-\mathbf{x}^{\prime}\right)\right]} \\
& =\int \frac{d^{4} \tilde{k}}{(2 \pi)^{3}} \delta\left(\tilde{k}^{2}-m^{2}\right) e^{-i \tilde{k} \cdot\left(\tilde{x}-\tilde{x}^{\prime}\right)}, \quad(\mathrm{A} 3)
\end{aligned}
$$

where $\tilde{k} \equiv k^{\mu}, \tilde{k}^{2} \equiv\left(k_{\mu} k^{\mu}\right),(\tilde{k} \cdot \tilde{x}) \equiv\left(k_{\mu} x^{\mu}\right)$, and in the final expression we have made use of the relation

$$
\begin{aligned}
\left(\frac{1}{2 \omega}\right)\left[\delta\left(k_{0}-\omega\right)+\delta\left(k_{0}+\omega\right)\right] & =\delta\left(k_{0}^{2}-\omega^{2}\right) \\
& =\delta\left(\tilde{k}^{2}-m^{2}\right) .
\end{aligned}
$$

On using the integral representation of the $\delta$ function, we obtain that

$$
\begin{aligned}
G^{(1)}\left(\tilde{x}, \tilde{x}^{\prime}\right) & =\int_{-\infty}^{\infty} d s e^{-i m^{2} s} \int \frac{d^{4} \tilde{k}}{(2 \pi)^{4}} e^{i \tilde{k}^{2} s} e^{-i \tilde{k} \cdot\left(\tilde{x}-\tilde{x}^{\prime}\right)} \\
& =\int_{-\infty}^{\infty} d s e^{-i m^{2} s} K\left(\tilde{x}, \tilde{x}^{\prime} ; s\right)
\end{aligned}
$$

where in the last expression we have identified the integral over $\tilde{k}$ to be the path-integral kernel of a free particle in four dimensions.

\section{APPENDIX B: DETERMINING THE NORMALIZATION CONSTANT $N_{E}$}

In this appendix, we shall evaluate the normalization constant $N_{E}$ for the wave functions corresponding to the Hamiltonian $\mathrm{H}_{k}$ in de Sitter. Recall that, in a de Sitter background, for the initial conditions of our interest, we can set $M_{E}$ to zero in Eq. (23), so that the wave function $\chi_{E}$ is given by

$$
\chi_{E}(t)=N_{E} H_{\nu}^{(1)}\left(k \mathcal{H}^{-1} e^{-\mathcal{H} t}\right),
$$

On substituting this wave function in the left-hand side of Eq. (18), we get

$$
\begin{aligned}
& \mathcal{I}\left(E, E^{\prime}\right)=\left(N_{E} N_{E^{\prime}}^{*}\right) \int_{-\infty}^{\infty} d t H_{\nu}^{(1)}\left(k \mathcal{H}^{-1} e^{-\mathcal{H} t}\right) \\
& \times H_{\nu^{\prime}}^{(1) *}\left(k \mathcal{H}^{-1} e^{-\mathcal{H} t}\right) \\
&=\left(N_{E} N_{E^{\prime}}^{*}\right) \int_{0}^{\infty} \frac{d \eta}{\mathcal{H} \eta} H_{\nu}^{(1)}(k \eta) H_{\nu^{\prime}}^{(1) *}(k \eta),
\end{aligned}
$$


where, in the second expression, we have expressed the integral in terms of the new independent variable $\eta=$ $\left(\mathcal{H} \mathrm{e}^{\mathcal{H} t}\right)^{-1}$. Also, the quantity $\nu^{\prime}$ denotes the quantity $\nu$ [defined in Eq. (24)] corresponding to the energy $E^{\prime}$.

In order to establish the normalization condition (18) and arrive at the value of normalization constant $N_{E}$, we shall adopt the following procedure. We shall relate the Hankel functions appearing in the integral (B2) above to the MacDonald function $K_{\nu}$ and use the following standard integral (see, for example, Ref. [31], p. 693):

$$
\begin{aligned}
\int_{0}^{\infty} d u u^{-\lambda} K_{\alpha}(p u) K_{\beta}(q u)= & \left(\frac{2^{-(2+\lambda)}}{\Gamma(1-\lambda)}\right) p^{-(1+\beta-\lambda)} q^{\beta} \\
& \times \Gamma\left(\frac{1-\lambda+\alpha+\beta}{2}\right) \Gamma\left(\frac{1-\lambda-\alpha-\beta}{2}\right) \Gamma\left(\frac{1-\lambda-\alpha+\beta}{2}\right) \Gamma\left(\frac{1-\lambda+\alpha-\beta}{2}\right) \\
& \times F\left[\left(\frac{1-\lambda+\alpha+\beta}{2}\right),\left(\frac{1-\lambda-\alpha+\beta}{2}\right),(1-\lambda),\left(\frac{a^{2}-b^{2}}{a^{2}}\right)\right],
\end{aligned}
$$

where $\Gamma$ denotes the Gamma function and $F$ is the hypergeometric function.

Note that, while the argument of the Hankel function appearing in the integral (B2) is always real, the index $\nu$ is real for $E<\left(9 H^{2} / 4\right)$, but it is imaginary for $E>\left(9 H^{2} / 4\right)$. For real values of the index, the Hankel functions $H_{\nu}^{(1)}$ and $H_{\nu}^{(2)}$ are complex conjugates of each other, and they are related to the MacDonald functions $K_{\nu}$ as follows (cf. Ref. [32], p. 375)

$$
\begin{aligned}
& H_{\nu}^{(1)}(z)=-\left(\frac{2 i}{\pi}\right) e^{-(i \pi \nu / 2)} K_{\nu}(-i z), \\
& H_{\nu}^{(2)}(z)=\left(\frac{2 i}{\pi}\right) e^{(i \pi \nu / 2)} K_{\nu}(i z) .
\end{aligned}
$$

Whereas, when $\nu$ is imaginary, the corresponding relations can be obtained to be

$$
\begin{aligned}
& H_{i|\nu|}^{(1)}(z)=-\left(\frac{2 i}{\pi}\right) e^{(\pi|\nu| / 2)} K_{i|\nu|}(-i z), \\
& H_{i|\nu|}^{(2)}(z)=\left(\frac{2 i}{\pi}\right) e^{-(\pi|\nu| / 2)} K_{i|\nu|}(i z) .
\end{aligned}
$$

For an imaginary index, the two Hankel functions are not complex conjugates of each other, but are related as follows:

$$
H_{i|\nu|}^{(1) *}(z)=e^{(\pi \nu)} H_{i|\nu|}^{(2)}(z)
$$

Let us first consider the case wherein $E$ and $E^{\prime}$ are both greater than $\left(9 H^{2} / 4\right)$ so that $\nu$ and $\nu^{\prime}$ are both imaginary. In such a case, on using the relations (B5a) and (B6), the integral (B2) can be expressed as

$$
\begin{aligned}
\mathcal{I}\left(E, E^{\prime}\right)= & \left(\frac{4 N_{E} N_{E^{\prime}}^{*}}{\pi^{2} \mathcal{H}}\right) e^{\left[\pi\left(|\nu|+\left|\nu^{\prime}\right|\right) / 2\right]} \\
& \times \int_{0}^{\infty} \frac{d \eta}{\eta} K_{i|\nu|}(-i k \eta) K_{i\left|\nu^{\prime}\right|}(i k \eta) .
\end{aligned}
$$

In order to identify the divergent part in the limit of $E \rightarrow E^{\prime}$, we shall express this integral as a limit in the following fashion:

$$
\begin{aligned}
\mathcal{I}(E, & \left.E^{\prime}\right) \\
= & \left(\frac{4 N_{E} N_{E^{\prime}}^{*}}{\pi^{2} \mathcal{H}}\right) e^{\left[\pi\left(|\nu|+\left|\nu^{\prime}\right|\right) / 2\right]} \\
& \times \lim _{\epsilon \rightarrow 0} \int_{0}^{\infty} d \eta \eta^{-(1-2 \epsilon)} K_{i|\nu|}(-i k \eta) K_{i\left|\nu^{\prime}\right|}(i k \eta) .
\end{aligned}
$$

On comparing Eqs. (B8) and (B3), we can identify that

$$
\begin{array}{rlrl}
\lambda & =(1-2 \epsilon), & \\
p=-(i k), & q=(i k), \\
\alpha & =(i|\nu|), & \beta=\left(i\left|\nu^{\prime}\right|\right),
\end{array}
$$

so that we have

$$
\begin{aligned}
\mathcal{I}\left(E, E^{\prime}\right)=\left(\frac{N_{E} N_{E^{\prime}}^{*}}{2 \pi^{2} \mathcal{H}}\right) e^{\left[\pi\left(|\nu|+\left|\nu^{\prime}\right|\right) / 2\right]} e^{\left(\pi \nu^{\prime}\right)} \Gamma\left[\frac{i\left(|\nu|+\left|\nu^{\prime}\right|\right)}{2}\right] \Gamma\left[\frac{-i\left(|\nu|+\left|\nu^{\prime}\right|\right)}{2}\right] & \\
& \times \lim _{\epsilon \rightarrow 0}\left[\frac{\Gamma\left(\epsilon+\left[i\left(|\nu|-\left|\nu^{\prime}\right|\right) / 2\right]\right) \Gamma\left(\epsilon-\left[i\left(|\nu|-\left|\nu^{\prime}\right|\right) / 2\right]\right)}{\Gamma(2 \epsilon)}\right]
\end{aligned}
$$


where we have made use of the fact that $F(a, b, c, 0)=1$ (cf. Ref. [32], p. 556). On using the following, standard properties of $\Gamma$ function (cf. Ref. [32], p. 256)

$$
\begin{aligned}
\Gamma(1+z) & =z \Gamma(z), \\
\Gamma(z) \Gamma(1-z) & =-z \Gamma(z) \Gamma(-z) \\
& =\left(\frac{\pi}{\sin (\pi z)}\right), \\
|\Gamma(i z)|^{2} & =\left(\frac{\pi}{z \sinh (\pi z)}\right), \\
|\Gamma(1+i z)|^{2} & =\left(\frac{\pi z}{\sinh (\pi z)}\right),
\end{aligned}
$$

we find that the expression (B10) can be expressed as

$$
\begin{aligned}
\mathcal{I}\left(E, E^{\prime}\right) & \\
=\left(\frac{N_{E} N_{E^{\prime}}^{*}}{\pi \mathcal{H}}\right) & \left(\frac{e^{\left[\pi\left(|\nu|+3\left|\nu^{\prime}\right|\right) / 2\right]}}{\left[\left(|\nu|+\left|\nu^{\prime}\right|\right) / 2\right] \sinh \left[\pi\left(|\nu|+\left|\nu^{\prime}\right|\right) / 2\right]}\right) \\
& \times\left(\frac{\left[\pi\left(|\nu|-\left|\nu^{\prime}\right|\right) / 2\right]}{\sinh \left[\pi\left(|\nu|-\left|\nu^{\prime}\right|\right) / 2\right]}\right) \\
& \times \lim _{\epsilon \rightarrow 0}\left(\frac{\epsilon}{\epsilon^{2}+\left[\left(|\nu|-\left|\nu^{\prime}\right|\right) / 2\right]^{2}}\right) \cdot(\mathrm{B} 12)
\end{aligned}
$$

Using the following representation of the delta function

$$
\delta(x)=\left(\frac{1}{\pi}\right) \lim _{\epsilon \rightarrow 0}\left(\frac{\epsilon}{\epsilon^{2}+x^{2}}\right),
$$

we can then write that

$$
\mathcal{I}\left(E, E^{\prime}\right)=\left(\frac{2\left|N_{E}\right|^{2}}{\mathcal{H}}\right)\left(\frac{e^{(2 \pi|\nu|)}}{|\nu| \sinh (\pi|\nu|)}\right) \delta\left(|\nu|-\left|\nu^{\prime}\right|\right) .
$$

From the relation between $E$ and $\nu$ [cf. Eq. (24)], we find that

$$
\begin{aligned}
\delta\left(E-E^{\prime}\right) & =\left(\frac{1}{\mathcal{H}^{2}}\right) \delta\left(\nu^{2}-\nu^{\prime 2}\right) \\
& =\left(\frac{1}{2 \mathcal{H}^{2}|\nu|}\right) \delta\left(|\nu|-\left|\nu^{\prime}\right|\right) .
\end{aligned}
$$

Therefore, we have

$$
\mathcal{I}\left(E, E^{\prime}\right)=\left(4 \mathcal{H}\left|N_{E}\right|^{2}\right)\left(\frac{e^{(2 \pi|\nu|)}}{\sinh (\pi|\nu|)}\right) \delta\left(E-E^{\prime}\right)
$$

which, finally, leads to

$$
\left|N_{E}\right|^{2}=\left(\frac{1}{4 \mathcal{H}}\right) \sinh (\pi|\nu|) e^{-(2 \pi|\nu|)} .
$$

Let us now assume that both $E$ and $E^{\prime}$ are less that $\left(9 H^{2} / 4\right)$ so that $\nu$ as well as $\nu^{\prime}$ are real quantities. As we had done earlier, we shall express the integral (B2) as a limit as follows:

$$
\begin{aligned}
\mathcal{I}\left(E, E^{\prime}\right)= & \left(\frac{4 N_{E} N_{E^{\prime}}^{*}}{\pi^{2} \mathcal{H}}\right) e^{-\left[i \pi\left(\nu-\nu^{\prime}\right) / 2\right]} \\
& \times \int_{0}^{\infty} d \eta \eta^{-(1-2 i \epsilon)} K_{\nu}(-i k \eta) K_{\nu^{\prime}}(i k \eta) .
\end{aligned}
$$

where we have made use of the relations (B4). On comparing Eqs. (B18) and (B3), we can identify that

$$
\lambda=(1-2 i \epsilon), a=-(i k), \quad b=(i k), \alpha=\nu \text { and } \beta=\nu^{\prime},
$$

so that we can write

$$
\begin{aligned}
\mathcal{I}\left(E, E^{\prime}\right)=\left(\frac{N_{E} N_{E^{\prime}}^{*}}{2 \pi^{2} \mathcal{H}}\right) e^{-\left[i \pi\left(\nu-3 \nu^{\prime}\right) / 2\right]} \Gamma\left[\frac{\nu+\nu^{\prime}}{2}\right] & \Gamma\left[-\left(\frac{\nu+\nu^{\prime}}{2}\right)\right] \\
& \times \lim _{\epsilon \rightarrow 0}\left[\frac{\Gamma\left(i \epsilon+\left[\left(\nu-\nu^{\prime}\right) / 2\right]\right) \Gamma\left(i \epsilon-\left[\left(\nu-\nu^{\prime}\right) / 2\right]\right)}{\Gamma(2 i \epsilon)}\right],
\end{aligned}
$$

where, again, we have made use of the result that $F(a, b, c, 0)=1$. On using the properties (B11) of the $\Gamma$ function, we find that the above expression can be written as

$$
\begin{aligned}
\mathcal{I}\left(E, E^{\prime}\right)= & \left(\frac{N_{E} N_{E^{\prime}}^{*}}{\pi \mathcal{H}}\right)\left(\frac{i e^{-\left[i \pi\left(\nu+3 \nu^{\prime}\right) / 2\right]}}{\left[\left(\nu+\nu^{\prime}\right) / 2\right] \sin \left[\pi\left(\nu+\nu^{\prime}\right) / 2\right]}\right) \\
& \times \Gamma\left(1+\left[\left(\nu-\nu^{\prime}\right) / 2\right]\right) \Gamma\left(1-\left[\left(\nu-\nu^{\prime}\right) / 2\right]\right) \times \lim _{\epsilon \rightarrow 0}\left(\frac{\epsilon}{\epsilon^{2}+\left[\left(\nu-\nu^{\prime}\right) / 2\right]^{2}}\right) \\
= & \left(\frac{2\left|N_{E}\right|^{2}}{\mathcal{H}}\right)\left(\frac{i e^{-(2 i \pi \nu)}}{\nu \sin (\pi \nu)}\right) \delta\left(\nu-\nu^{\prime}\right)=\left(4 \mathcal{H}\left|N_{E}\right|^{2}\right)\left(\frac{i e^{-(2 i \pi \nu)}}{\nu \sin (\pi \nu)}\right) \delta\left(E-E^{\prime}\right),
\end{aligned}
$$

where in arriving at the second and the third equalities, we have made use of the relations (B13) and (B15), re- spectively. Dropping irrelevant phase factors, we obtain

$$
\left|N_{E}\right|^{2}=\left(\frac{1}{4 \mathcal{H}}\right)|\sin (\pi \nu)|
$$


We have quoted the results (B17) and (B22) in Eq. (26).

\section{APPENDIX C: PATH INTEGRAL DUALITY MODIFIED 'DENSITY OF STATES'}

In this appendix, we outline the derivation of the result we have quoted in Eq. (32). Let

$$
\mathcal{G}\left(E, L_{\mathrm{P}}\right)=\int_{-\infty}^{\infty} d s e^{i\left[\left(L_{\mathrm{P}}^{2} / s\right)+E s\right]}
$$

which can be expressed as

$$
\begin{aligned}
\mathcal{G}\left(E, L_{\mathrm{P}}\right)= & \int_{0}^{\infty} d s e^{i\left[\left(L_{\mathrm{P}}^{2} / s\right)+E s\right]}+\int_{0}^{\infty} d s e^{-i\left[\left(L_{\mathrm{P}}^{2} / s\right)+E s\right]} \\
= & \lim _{\epsilon \rightarrow 0^{+}} \int_{0}^{\infty} d s e^{-\left[(\epsilon-i E) s-\left(i L_{\mathrm{P}}^{2} / s\right)\right]} \\
& +\lim _{\epsilon \rightarrow 0^{+}} \int_{0}^{\infty} d s e^{-\left[(\epsilon+i E) s+\left(i L_{\mathrm{P}}^{2} / s\right)\right]}, \quad(\mathrm{C} 2)
\end{aligned}
$$

where we have introduced a small parameter $\epsilon$ which we shall eventually set to zero. On using the following integral representation of the modified Bessel function (cf. Ref. [31], p. 376)

$$
\int_{0}^{\infty} d u \exp -[a u+(b / u)]=\sqrt{\frac{4 b}{a}} K_{1}(\sqrt{4 a b})
$$

which is valid for Re. $a>0$ and Re. $(b) \geq 0$, we obtain that

$$
\begin{aligned}
& \mathcal{G}\left(E, L_{\mathrm{P}}\right) \\
& =\lim _{\epsilon \rightarrow 0^{+}} 2\left(\frac{-i L_{\mathrm{P}}^{2}}{\epsilon-i E}\right)^{1 / 2} K_{1}\left(2\left[-i L_{\mathrm{P}}^{2}(\epsilon-i E)\right]^{1 / 2}\right) \\
& +\lim _{\epsilon \rightarrow 0^{+}} 2\left(\frac{i L_{\mathrm{P}}^{2}}{\epsilon+i E}\right)^{1 / 2} K_{1}\left(2\left[i L_{\mathrm{P}}^{2}(\epsilon+i E)\right]^{1 / 2}\right),
\end{aligned}
$$

We shall now utilize the following series representation of the Macdonald function $K_{1}$ (cf. Ref. [32], p. 375):

$$
\begin{aligned}
K_{1}(z)= & \left(\frac{1}{z}\right)+\ln \left(\frac{z}{2}\right) I_{1}(z) \\
& -\left(\frac{z}{4}\right) \sum_{k=0}^{\infty}\left(\frac{\psi(k+1)+\psi(k+2)}{k !(k+1) !}\right)\left(\frac{z}{2}\right)^{2 k},
\end{aligned}
$$

where $I_{1}$ is the modified Bessel function of order unity and $\psi$ denotes the di-gamma function. On using this series representation and the relation $I_{1}(z)=-\left[i J_{1}(i z)\right]$
( $J_{1}$ being the Bessel function), we find that we can write the modified 'density of states' as follows:

$$
\begin{aligned}
\mathcal{G}\left(E, L_{\mathrm{P}}\right) & =\lim _{\epsilon \rightarrow 0^{+}}\left(\frac{2 \epsilon}{\epsilon^{2}+E^{2}}\right)+\left(\frac{2 \pi i L_{\mathrm{P}}}{\sqrt{E}}\right) I_{1}\left(2 i L_{\mathrm{P}} \sqrt{E}\right) \\
& =(2 \pi)\left[\delta(E)-\left(\frac{L_{P}}{\sqrt{E}}\right) J_{1}\left(2 L_{\mathrm{P}} \sqrt{E}\right)\right], \quad(\mathrm{C} 6)
\end{aligned}
$$

where we have also made use of the representation (B13) of the delta function. This is the result (32) we have made use in the text.

\section{APPENDIX D: A CONSISTENCY CHECK-POWER SPECTRUM IN THE LIMIT OF $\mathcal{H} \rightarrow 0$}

As a consistency check, in this appendix, we shall explicitly show that, in the limit of $H \rightarrow 0$, the modified power spectrum in de Sitter reduces to that of the corresponding modified spectrum for a scalar field in flat space-time. In order to do so, let us first evaluate the modified spectrum in flat space-time.

In flat space-time (i.e. when $a=1$ ), the normalized wave functions corresponding to the Schrodinger equation (14) are given by

$$
\chi_{E}(t)=\left(\frac{1}{4 \pi \sqrt{k^{2}+E}}\right)^{1 / 2} e^{-i \sqrt{k^{2}+E} t} .
$$

On subsituting this wave function in the expression (33) for the modified power spectrum, we obtain that

$$
\begin{aligned}
& {\left[k^{3} \mathcal{P}_{\Phi}(k)\right]_{(\mathrm{M})}} \\
& \quad=\left(\frac{k^{2}}{8 \pi^{2}}\right)-\left(\frac{L_{\mathrm{P}} k^{3}}{8 \pi^{2}}\right) \int_{0}^{\infty} \frac{d E}{\sqrt{E\left(k^{2}+E\right)}} J_{1}\left(2 L_{\mathrm{P}} \sqrt{E}\right) \\
& \quad=\left(\frac{k^{2}}{8 \pi^{2}}\right)\left[1-2 e^{-k L_{\mathrm{P}}} \sinh \left(k L_{\mathrm{P}}\right)\right] \\
& =\left(\frac{k^{2}}{8 \pi^{2}}\right) e^{-2 k L_{\mathrm{P}}}
\end{aligned}
$$

This expression illustrates the fact that the duality principle leads to an exponential suppression of power for the trans-Planckian modes (i.e. modes for which $k \gg L_{\mathrm{P}}^{-1}$ ).

We shall now outline as to how the modified power spectrum in exponential inflation reduces to the above flat space-time expression in the limit of $\mathcal{H} \rightarrow 0$. In this limit, we find that the wave function (27) in de Sitter space reduces to

$$
\begin{aligned}
\chi_{E}(t) \simeq(4 \mathcal{H})^{-1 / 2} & e^{-(\pi \sqrt{E} / 2 \mathcal{H})} \\
& \times H_{(i \sqrt{E} / \mathcal{H})}^{(1)}\left(k \mathcal{H}^{-1} e^{-\mathcal{H} t}\right) .
\end{aligned}
$$

Also, these wave functions are valid in the entire energy range of $0<E<\infty$. For large complex order and real 
argument, the asymptotic form of the Hankel function $H_{i \nu}^{(1)}(z)$ is given by (cf. Ref. [35], p. 263)

$$
H_{i \nu}^{(1)}(z) \simeq[-(\pi \nu i / 2) \tanh \gamma]^{-1 / 2} e^{\nu(\tanh \gamma-\gamma)} e^{-i \pi / 4}
$$

where $\nu=(z \cosh \gamma), \gamma=(\alpha+i \beta)$ and $0<\beta<\pi$. If we choose $\beta=(\pi / 2)$, then, we find that $\nu=(i z \sinh \alpha)$ and $\tanh \gamma=\operatorname{coth} \alpha$. In our case, we have $\nu=(i \sqrt{E} / H), z=$ $\left(k \mathcal{H}^{-1} e^{-\mathcal{H} t}\right)$ so that $\sinh \alpha=\left(\sqrt{E} k^{-1} e^{-\mathcal{H} t}\right)$. On using these expressions in the above asymptotic expansion of the Hankel function, we find that, as $\mathcal{H} \rightarrow 0$,

$$
\left|H_{(i \sqrt{E} / \mathcal{H})}^{(1)}\left(k \mathcal{H}^{-1} e^{-\mathcal{H} t}\right)\right|^{2} \rightarrow\left(\frac{\mathcal{H} \tanh \alpha}{\pi \sqrt{E}}\right) e^{(\pi \sqrt{E} / \mathcal{H})}=\left(\frac{\mathcal{H}}{\pi \sqrt{k^{2}+E}}\right) e^{(\pi \sqrt{E} / \mathcal{H})}
$$

so that we have

$$
\left|\chi_{E}(t)\right|^{2} \simeq\left(\frac{1}{4 \pi \sqrt{k^{2}+E}}\right)
$$

which is the amplitude of the wave function in flat spacetime. Evidently, substituting this wave function in the expression (33) for the modified spectrum will lead to the required spectrum in flat space-time.

It should be emphasized here that we have arrived at the flat space-time result by taking the limit of $\mathcal{H} \rightarrow 0$ without imposing the condition of Hubble exit in the expression for the power spectrum in a de Sitter background. Physically, the limit of $\mathcal{H} \rightarrow 0$ is equivalent to sub-Hubble scales in exponential inflation and, therefore, this limit leads to the flat space-time result. It should be noted that the condition of Hubble exit and the limit $\mathcal{H} \rightarrow 0$ correspond to two extreme limits. Hence, we could not have arrived at the flat space-time result by setting $\mathcal{H}=0$ in the power spectrum evaluated at Hubble exit in exponential inflation.
[1] A. D. Linde, Particle Physics and Inflationary Cosmology (Harwood Academic, Chur, Switzerland, 1990); A. R. Liddle and D. H. Lyth, Cosmological Inflation and Large-Scale Structure (Cambridge University Press, Cambridge, England, 1999); V. F. Mukhanov, Physical Foundations of Cosmology, (Cambridge University Press, Cambridge, England, 2005).

[2] V. F. Mukhanov, H. A. Feldman and R. H. Brandenberger, Phys. Rep. 215, 203 (1992).

[3] J. E. Lidsey, A. Liddle, E. W. Kolb, E. J. Copeland, T. Barreiro and M. Abney, Rev. Mod. Phys. 69, 373 (1997).

[4] B. Bassett, S. Tsujikawa and D. Wands, Rev. Mod. Phys. 78, 537 (2006).

[5] D. L. Lyth and A. Riotto, Phys. Rep. 314, 1 (1999).

[6] T. Padmanabhan, Phys. Rev. Letts. 602229 (1988); T. Padmanabhan, T. R. Seshadri and T. P. Singh, Phys. Rev. D 39, 2100 (1989).

[7] R. Brandenberger and J. Martin, Mod. Phys. Lett. A 16, 999 (2001); J. Martin and R. Brandenberger, Phys. Rev. D 63, 123501 (2001); J. C. Niemeyer, Phys. Rev. D 63, 123502 (2001); M. Lemoine, M. Lubo, J. Martin and J. P. Uzan, Phys. Rev. D 65, 023510 (2002); J. Martin and R. Brandenberger, Phys. Rev. D 65, 103514 (2002); R. Brandenberger and J. Martin, Int. J. Mod. Phys. A 17, 3663 (2002); J. Martin and R. Brandenberger, Phys. Rev. D 68, 063513 (2003).

[8] S. Shankaranarayanan and M. Lubo, Phys. Rev. D 72, 123513 (2005).

[9] A. A. Starobinsky, Pisma Zh. Eksp. Teor. Fiz. 73, 415 (2001); S. Shankaranaryanan, Class. Quantum Grav. 20,
75 (2003); J. C. Niemeyer and R. Parentani, Phys. Rev. D 64, 101301 (2001).

[10] A. Kempf and J. C. Niemeyer, Phys. Rev. D 64, 103501 (2001); R. Easther, B. R. Greene, W. H. Kinney and G. Shiu, Phys. Rev. D 64, 103502 (2001); R. Easther, B. R. Greene, W. H. Kinney and G. Shiu, Phys. Rev. D 66, 023518 (2002); S. F. Hassan and M. S. Sloth, Nucl. Phys. B 674, 434 (2003).

[11] C. S. Chu, B. R. Greene and G. Shiu, Mod. Phys. Lett. A 16, 2231 (2001); F. Lizzi, G. Mangano, G. Miele and M. Peloso, JHEP 0206, 049 (2002); R. Brandenberger and P. M. Ho, Phys. Rev. D 66, 023517 (2002).

[12] U. H. Danielsson, Phys. Rev. D 66, 023511 (2002); U. H. Danielsson, JHEP 0212, 025 (2002); U. H. Danielsson, D. Domert and M. Olsson, Phys. Rev. D 68, 083508 (2003)

[13] N. Kaloper, M. Kleban, A. E. Lawrence and S. Shenker, Phys. Rev. D 66, 123510 (2002).

[14] S. Shankaranarayanan and L. Sriramkumar, Phys. Rev. D 70, 123520 (2004).

[15] C. P. Burgess, J. M. Cline, F. Lemieux and R. Holman, JHEP 0302, 048 (2003).

[16] L. Sriramkumar and T. Padmanabhan, Phys. Rev. D 71, 103512 (2005).

[17] L. Bergstrom and U. H. Danielsson, arXiv:hepth/0211006.

[18] Q. G. Huang and M. Li, JHEP 0306, 014 (2003); S. Tsujikawa, R. Maartens and R. Brandenberger, Phys. Lett. B 574, 141 (2003);

[19] S. Tsujikawa, P. Singh and R. Maartens, arXiv:astro$\mathrm{ph} / 0311015$. 
[20] J. Martin and C. Ringeval, Phys. Rev. D 69, 083515 (2004); R. Easther, W. H. Kinney and H. Peiris, JCAP 0505, 009 (2005); R. Easther, W. H. Kinney and H. Peiris, JCAP 0508, 001 (2005).

[21] T. Jacobson, S. Liberati and D. Mattingly, Nature 424, 1019 (2003); R. C. Myers and M. Pospelov, Phys. Rev. Lett. 90, 211601 (2003).

[22] G. Amelino-Camelia, arXiv:astro-ph/0410076.

[23] T. Padmanabhan, Phys. Rev. Letts. 78, 1854 (1997); T. Padmanabhan, Phys. Rev. D 57, 6206 (1998).

[24] P. R. Chouha and R. H. Brandenberger, arXiv:hepth/0508119.

[25] A. Smailagic, E. Spallucci and T. Padmanabhan, arXiv:hep-th/0308122; M. Fontanini, E. Spallucci and T. Padmanabhan, Phys. Lett. B 633, 627 (2006).

[26] J. Schwinger, Phys. Rev. 82 (1951), 664.

[27] B. S. DeWitt, Phys. Rep. 19 (1975), 297.
[28] I. H. Duru and N. Unal, Phys. Rev. D 34, 959 (1986).

[29] R. P. Feynman and A. R. Hibbs, Quantum Mechanics and Path Integrals (McGraw-Hill, Singapore, 1965).

[30] L. S. Schulman, Techniques and Applications of Path Integration (John Wiley, New York, 1981).

[31] I. S. Gradshteyn and I. M. Ryzhik, Table of Integrals, Series and Products (Academic, New York, 1980).

[32] M. Abramowitz and I. A. Stegun, Handbook of Mathematical Functions (Dover Publications, New York, 1964).

[33] T. Bunch and P. C. W. Davies, Proc. Roy. Soc. Lond. A 360, 117 (1978).

[34] G. M. Hossain, Class. Quant. Grav. 22, 2511 (2005); S. Hofmann and O. Winkler, arXiv:astro-ph/0411124.

[35] G. N. Watson, A Treatise on the Theory of Bessel functions (Cambridge University Press, Cambridge, England, 1995). 